\title{
Status report on the Large Binocular Telescope's ARGOS ground-layer AO system
}

\author{
M. Hart, ${ }^{1}$ S. Rabien, ${ }^{2}$ L. Busoni, ${ }^{3}$ L. Barl, ${ }^{2}$ U. Beckmann, ${ }^{5}$ M. Bonaglia, ${ }^{3}$ Y. Boose,,${ }^{2}$ J. L. Borelli, ${ }^{4}$ T. \\ Bluemchen, ${ }^{4}$ L. Carbonaro, ${ }^{3}$ C. Connot, ${ }^{5}$ M. Deysenroth, ${ }^{2}$ R. Davies, ${ }^{2}$ O. Durney, ${ }^{1}$ M. Elberich, ${ }^{5}$ T. \\ Ertl, ${ }^{2}$ S. Esposito, ${ }^{3}$ W. Gaessler, ${ }^{4}$ V. Gasho, ${ }^{1}$ H. Gemperlein, ${ }^{2}$ P. Hubbard, ${ }^{1}$ S. Kanneganti, ${ }^{2}$ M. \\ Kulas, ${ }^{4}$ K. Newman, ${ }^{1}$ J. Noenickx, ${ }^{1}$ G. Orban de Xivry, ${ }^{2}$ D. Peter, ${ }^{4}$ A. Quirrenbach, ${ }^{6}$ M. \\ Rademacher, ${ }^{1}$ C. Schwab, ${ }^{6}$ J. Storm, ${ }^{7}$ V. Vaitheeswaran, ${ }^{1}$ G. Weigelt, ${ }^{5}$ J. Ziegleder ${ }^{2}$ \\ ${ }^{1}$ Steward Observatory, University of Arizona, Tucson, AZ 85721, U.S.A. \\ ${ }^{2}$ Max-Planck-Institut für extraterrestrische Physik, 85748 Garching, Germany \\ ${ }^{3}$ Osservatorio Astrofisico di Arcetri, Largo E. Fermi 5, I-50125 Firenze, Italy \\ ${ }^{4}$ Max-Planck-Institut für Astronomie, D-69117 Heidelberg, Germany \\ ${ }^{5}$ Max-Planck-Institut für Radioastronomie, 53121 Bonn, Germany \\ ${ }^{6}$ Landessternwarte, D-69117 Heidelberg, Germany \\ ${ }^{7}$ Astrophysikalisches Institut D-14482 Potsdam, Germany
}

\begin{abstract}
ARGOS, the laser-guided adaptive optics system for the Large Binocular Telescope (LBT), is now under construction at the telescope. By correcting atmospheric turbulence close to the telescope, the system is designed to deliver high resolution near infrared images over a field of 4 arc minute diameter. Each side of the LBT is being equipped with three Rayleigh laser guide stars derived from six $18 \mathrm{~W}$ pulsed green lasers and projected into two triangular constellations matching the size of the corrected field. The returning light is to be detected by wavefront sensors that are range gated within the seeing-limited depth of focus of the telescope. Wavefront correction will be introduced by the telescope's deformable secondary mirrors driven on the basis of the average wavefront errors computed from the respective guide star constellation. Measured atmospheric turbulence profiles from the site lead us to expect that by compensating the ground-layer turbulence, ARGOS will deliver median image quality of about $0.2 \mathrm{arcsec}$ across the JHK bands. This will be exploited by a pair of multi-object near-IR spectrographs, LUCIFER1 and LUCIFER2, with 4 arc minute field already operating on the telescope. In future, ARGOS will also feed two interferometric imaging instruments, the LBT Interferometer operating in the thermal infrared, and LINC-NIRVANA, operating at visible and near infrared wavelengths. Together, these instruments will offer very broad spectral coverage at the diffraction limit of the LBT's combined aperture, $23 \mathrm{~m}$ in size.
\end{abstract}

Keywords: Adaptive optics, Large Binocular Telescope, laser guide stars

\section{INTRODUCTION}

The Large Binocular Telescope (LBT), shown in Figure 1 offers uniquely powerful scientific capabilities. In particular the $22.8 \mathrm{~m}$ baseline provided by the two primary mirrors, when co-phased, will offer the highest spatial resolution for studies of faint objects, making the LBT arguably the forerunner of the next generation of Extremely Large Telescopes (ELTs). In common with other large telescopes around the world it relies on adaptive optics (AO) to deliver high resolution imaging and spectroscopy. In fact the LBT was designed from the outset to include AO as an integral part of the telescope. Uniquely among telescopes of $8 \mathrm{~m}$ and above, the AO correction is built in to the LBT's adaptive secondary mirrors (ASM). This capability allows for correction of all instruments used at the Gregorian foci, without any requirement for relay optics that introduce losses and increase thermal background, complexity, and cost. In addition, the deployment of Gregorian ASMs greatly facilitates the implementation of ground-layer AO (GLAO) by allowing a wide corrected field of view while simultaneously conjugating the wavefront corrector close to the locally induced aberration.

While first-light AO relies on natural guide stars (NGS), ${ }^{1,2,3}$ the LBT Observatory has launched a phased program to augment the telescope with laser guide stars. Phase I, called the Advanced Rayleigh Ground layer adaptive Optics

Astronomical Adaptive Optics Systems and Applications IV, edited by Robert K. Tyson, Michael Hart, Proc. of SPIE Vol. 8149, 81490J · C 2011 SPIE · CCC code: 0277-786X/11/\$18 · doi: 10.1117/12.893916 
System (ARGOS) is now in advanced development. The science requirements for the Phase I system lead to two principal goals:

- Enhance the JHK band image quality and ensquared energy by a factor 2-3 for the LUCIFER instruments.

- Implement a reliable, low maintenance and robust system with high observing efficiency.

ARGOS will achieve these goals through the deployment of six low-level Rayleigh LGS, three per aperture, to correct the lowlying turbulence that is isoplanatic over a wide field of view. Detailed site studies with SCIDAR $^{4,5}$ have shown that the LBT site manifests a boundary layer of turbulence which frequently accounts for the majority of the seeing; Figure 2 shows the median of the profiles in this study. Removing the effect of this layer with GLAO would offer image resolution in the near IR bands better than 0.2 arc sec over a field of $\sim 5 \operatorname{arc} \min$ as a

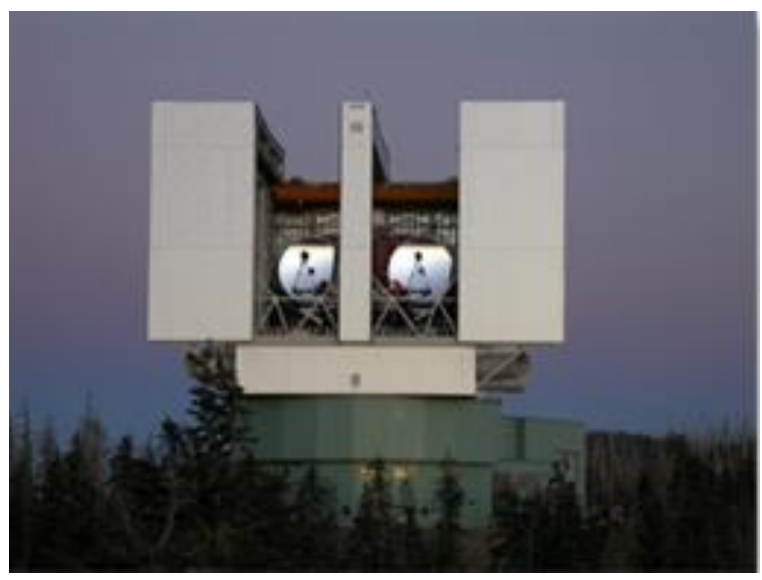

Figure 1. The Large Binocular Telescope on Mt. Graham, Arizona has two $8.4 \mathrm{~m}$ primary mirrors on a common mount. matter of routine. In this mode of operation, ARGOS will feed the 4 arc min wide-field modes of the LUCIFER near-infrared imagers and multi-object spectrographs with images that routinely reach $\sim 0.2-0.3 \mathrm{arc} \mathrm{sec}$ resolution. The Phase I system passed a Final Design Review in March 2010, and is on track for deployment as a facility system in 2012.

Phase II is to be developed concurrently. A sodium LGS will be added to each aperture using the same launch optics as the Rayleigh beacons. Additional wavefront sensors (WFS) will be deployed in front of the LUCIFERs with feedback to the ASMs. These instruments will then enjoy images sharpened to the diffraction limit of the individual $8.4 \mathrm{~m}$ apertures in the JHK wavebands.

Truly ground-breaking, however, will be the implementation of sodium LGS AO correction for the LBT Interferometer (LBTI). This instrument will bring together the beams from the two halves of the telescope in Fizeau mode, mimicking a filled pupil masked with two $8.4 \mathrm{~m}$ apertures. Imaging will be available with the full resolving power of the LBT with $22.8 \mathrm{~m}$ baseline at wavelengths from 2 to $20 \mu \mathrm{m}$, and with unique sensitivity in the thermal bands because of the minimal number of warm optics in the beam. High-quality AO control of the individual apertures will be provided by the sodium LGS and additional WFS placed in each arm of the LBTI, with piston control between the apertures provided by a Kband fringe tracker already built into the instrument. In addition, LBTI can be configured to operate as a Bracewell nulling interferometer, for which the laser $\mathrm{AO}$ will allow high-contrast investigation in the thermal IR of the environments of deeply embedded stars that are very faint in the optical.

In a further development, Phase III will combine use of the low-altitude Rayleigh and high-altitude sodium LGS into a uniquely powerful tomographic wavefront sensing system for multi-conjugate adaptive optics (MCAO). This hybrid sensing system will require just a single tip-tilt star for full multi-conjugate correction. Such a scheme overcomes a limitation of MCAO systems in which the beacons are all at a common range that leads to a requirement for three well separated tip-tilt stars. ${ }^{6,7}$ This is the case, for example, with the Gemini South MCAO system ${ }^{8}$ which uses five sodium LGS, but will be somewhat limited in its sky coverage by the need for multiple tip-tilt stars of magnitude $\sim 18$ or brighter within 1 arc min.

MCAO is already designed as an upgrade path into LBT's LINC-NIRVANA interferometer, ${ }^{4}$ now nearing completion at the Max-Planck Institute for Astronomy

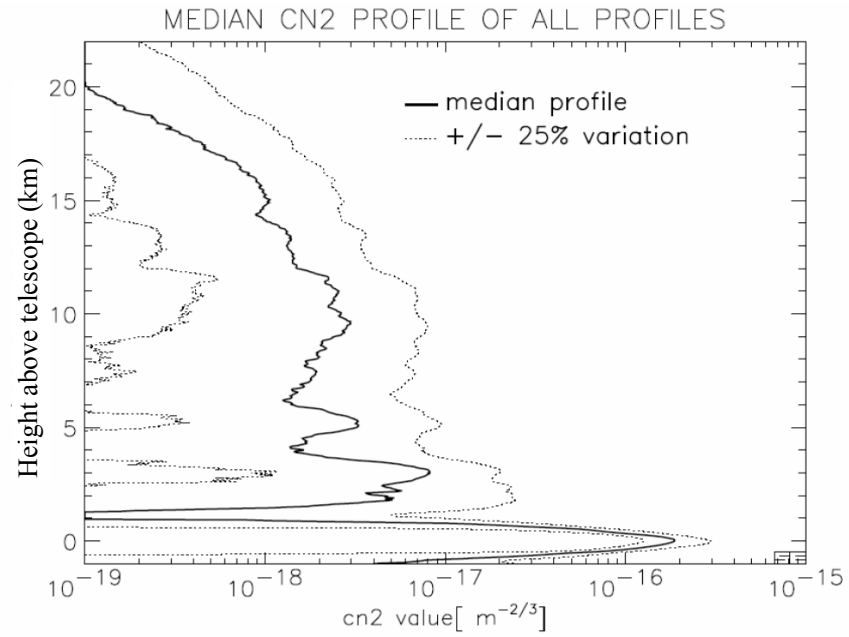

Figure 2. Median, 25, and 75 percentile $\mathrm{C}_{\mathrm{n}}{ }^{2}$ profiles derived from SCIDAR measurements at the LBT site. 
in Heidelberg as a collaboration between LBT's German and Italian partners. Expected to be operational in 2012, LINCNIRVANA will extend the full resolution of the coherently combined telescope apertures down to J band in the near IR with resolution as high as 10 milliarcsec. To give an example of its application, at this resolution, and with LBT's sensitivity, equivalent to a $12 \mathrm{~m}$ single aperture, stellar populations in galaxies at 5-20 Mpc will be resolved. For the first time, individual stars in giant elliptical galaxies will be within reach, allowing their star formation history to be investigated directly.

\section{OVERALL DESIGN OF ARGOS}

The ARGOS GLAO system relies on multiple low-altitude Rayleigh laser guide stars (RLGS) to assure uniform correction of the point-spread function (PSF) over the science field, up to 4 arc min square. The beacons are made with $532 \mathrm{~nm}$ light from frequency double Nd:YAG lasers that are pulsed at $10 \mathrm{kHz}$. Three beacons above each eye of the LBT are arranged in two equilateral triangles, clocked to align the returning beams conveniently with the optical axis of the LUCIFER instruments, the main science tool to be coupled to the GLAO system (Figure 3).

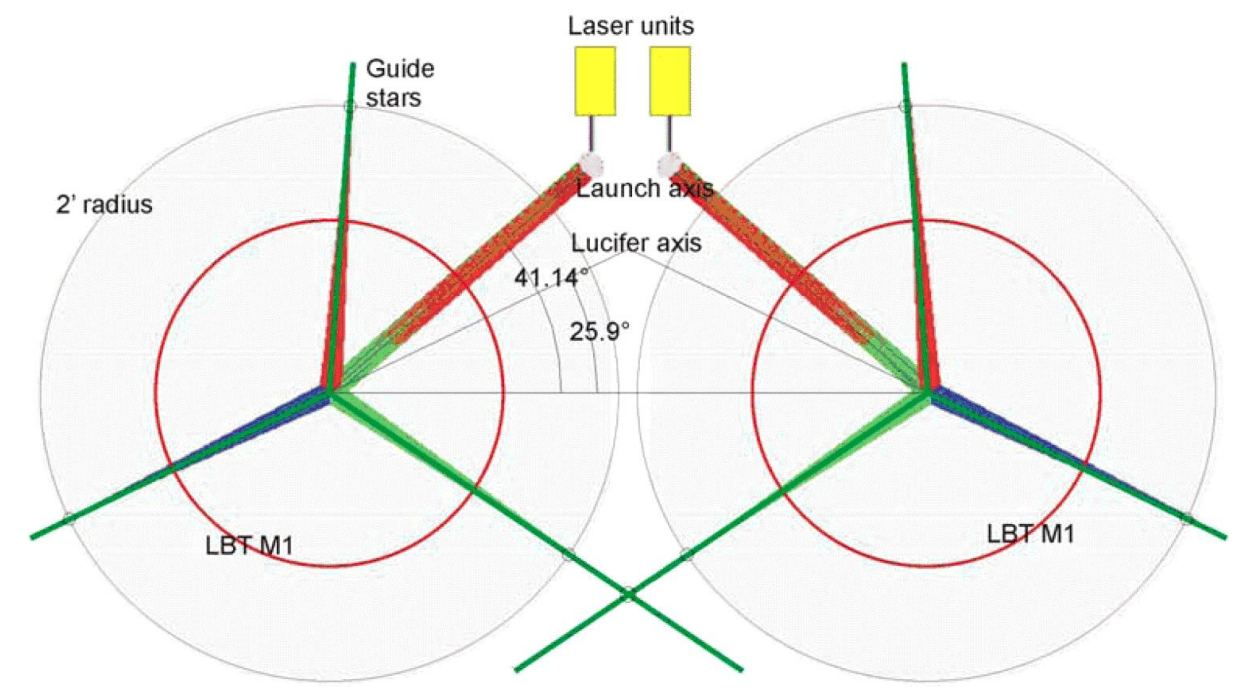

Figure 3. Looking down at the LBT from above, the six ARGOS laser beams are arranged as two equilateral triangles. The beacons themselves are put at a 2 arc min radius around the field of view.

Figure 4 shows a schematic overview of the telescope and the main component of the ARGOS system as they will be mounted. The lasers themselves are installed near the middle of the telescope structure, with refractive launch optics used to expand the beams and direct them to the sky from behind the telescope's two secondary mirrors. Back-scattered laser light returning to the telescope is intercepted in front of the LUCIFER instruments by a large notch reflector beam splitter that covers the full field of the instrument. Most of the incident light including science light in the near IR is transmitted, while the laser light is reflected to a WFS unit that houses the sensor itself as well as patrol cameras that can explore a 1 arc min field for the purpose of acquiring the RLGS. Pockels cells ahead of the WFS implement the required range gate to capture RLGS light only from the desired range of altitudes around $12 \mathrm{~km}$. A fast sensor implemented as a quad cell of avalanche photodiodes directly ahead of LUCIFER will use visible starlight transmitted by the beam splitter to sense overall image motion that is not recoverable from the laser signals.

A dedicated computer will calculate the wavefront slopes from the WFS signals. The slope vectors will then be transmitted for wavefront reconstruction and real-time ASM control to existing computers mounted in the secondary mirror hubs which already serve the purpose for the telescope's NGS AO system. In addition, the pyramid WFS of the NGS AO system will be used at a very slow frame rate, using a small fraction of the tilt starlight, to provide truth sensing. Offsets to the LGS WFS centroid zero points will be modified dynamically to remove any slowly drifting aberration that is observed in this way. The whole ARGOS edifice is operated by a software and electro-mechanical control system. ${ }^{9}$ 


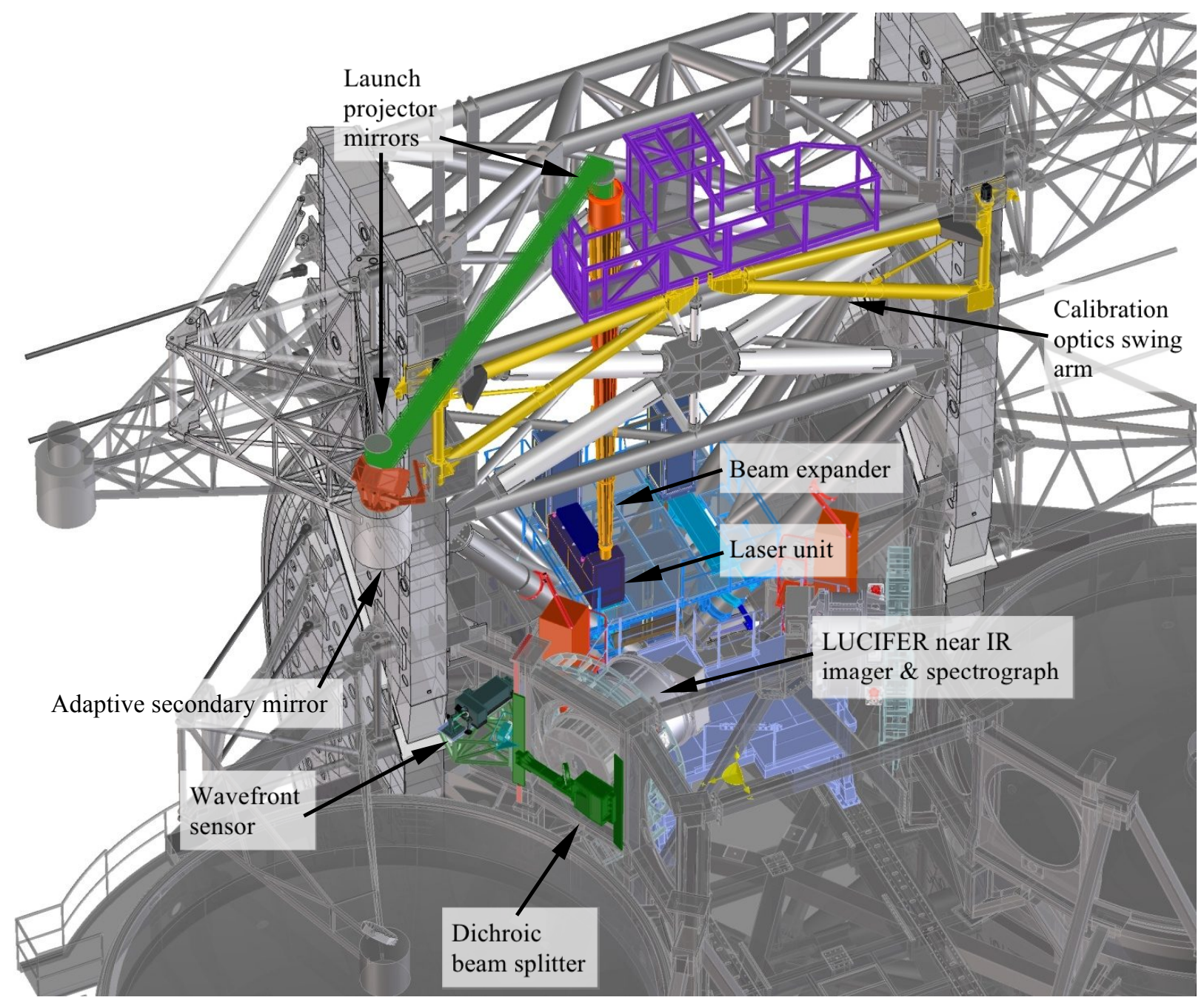

Figure 4. Overview of the ARGOS components as they are being installed at the LBT. The lasers themselves will be installed in the center one level above the primary mirrors. A dedicated platform allows access and servicing. The laser beams are launched through a refractive telescope on each side that expands them to a $40 \mathrm{~cm}$ exit pupil at an aspheric lens attached to the top of the LBT structure. The beams are directed by large flat mirrors into the sky from behind the secondary mirror. Returning laser light is separated from the science beam by a large dichroic mirror and sent to the wavefront sensor. Additional swing arms allow calibration light sources and optics to be moved into the Gregorian prime focus to calibrate the adaptive optics during daytime.

\section{LASER AND LAUNCH PROJECTOR}

The six lasers themselves are each $18 \mathrm{~W}$ pulsed frequency-doubled Nd:YAG units, and are now in hand. The pulse repetition rate is $10 \mathrm{kHz}$ with pulses of $<40 \mathrm{~ns}$. The laser heads will reside in two enclosed boxes, one on each side of the telescope, that will also hold the optics needed to condition the beams and arrange them into the constellation for projection. The layout is illustrated in Figure 5, where for clarity the enclosing sides of the box have been omitted.

The conditioning optics impose a linear polarization on the beams whose axis is chosen to match the preferred direction of the coating on the beam splitter ahead of the science instrument. Since Rayleigh scattering in the atmosphere preserves polarization, this ensures that the best system transmission is preserved, and also allows the use of Pockels cell switching in the WFS to implement the range gate. In addition, each laser box includes a safety shutter which, when closed, sends the beams to a power meter. Motorized periscopes allow the pointing of each of the three beams to be adjusted independently. Diverger lenses expand the beams to an exit pupil of $8 \mathrm{~mm}$ diameter on a fold flat that sends the 
light vertically up into the launch projector. This fold flat is mounted on a fast tip-tilt stage that serves to compensate jitter in the outgoing beam.

The launch telescopes each comprise a refractive beam expander followed by large fold mirrors to steer the beams so that they are directed into the sky from behind the two secondary mirrors of the LBT. Starting at the bottom, a small lens assembly inside the laser box diverges the beams at approximately $\mathrm{f} / 25$. Such a slow focal ratio allows loose alignment tolerances that can also accommodate the anticipated gravitational flexure of the telescope structure. The assembly rides on a longitudinal translation stage which provides the focus mechanism for the launch optics. Near the top of the LBT, a $45 \mathrm{~cm}$ diameter plano-convex singlet lens focuses the outgoing beams to a range of $12 \mathrm{~km}$ from the telescope. The convex side of the lens is aspheric to allow it to be mounted in an unconventional orientation, with the curved surface towards the diverging beam. In this way, the flat surface is downstream of all powered surfaces and will be used as a retroreflector to aid in interferometric alignment of the launch optics once mounted on the LBT. One of these two large lenses, manufactured by SESO, is shown mounted in its cell in Figure 6. Also shown is one of four identical borosilicate honeycomb fold flats that steer the full size beams across the top of the LBT. All the refractive and reflective optics are coated to assure the highest performance at both the $532 \mathrm{~nm}$ wavelength of the Rayleigh beacons and at $589 \mathrm{~nm}$, the wavelength of the anticipated expansion to include additional sodium LGS.
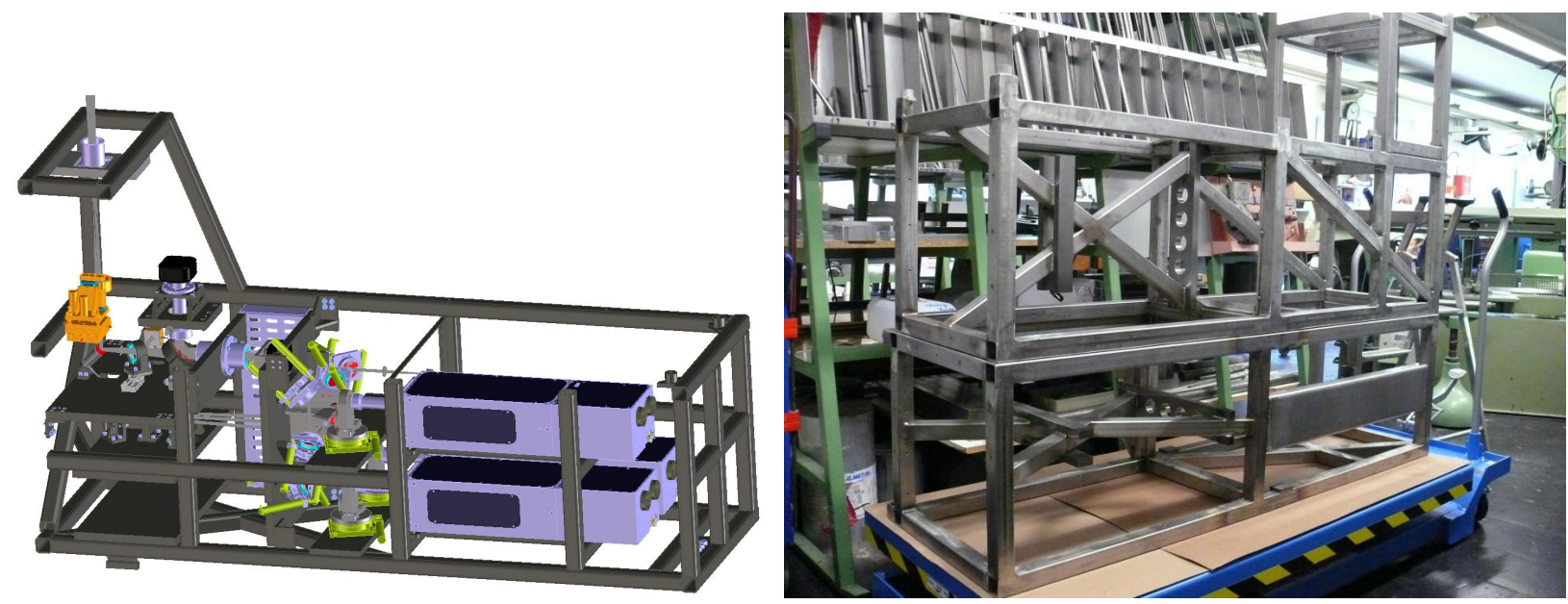

Figure 5. (Left) Solid model of the lasers and beam conditioning optics for one side of the telescope mounted in their steel frame. (Right) The two frames welded and ready for tempering. Each frame is about $2 \mathrm{~m}$ long.
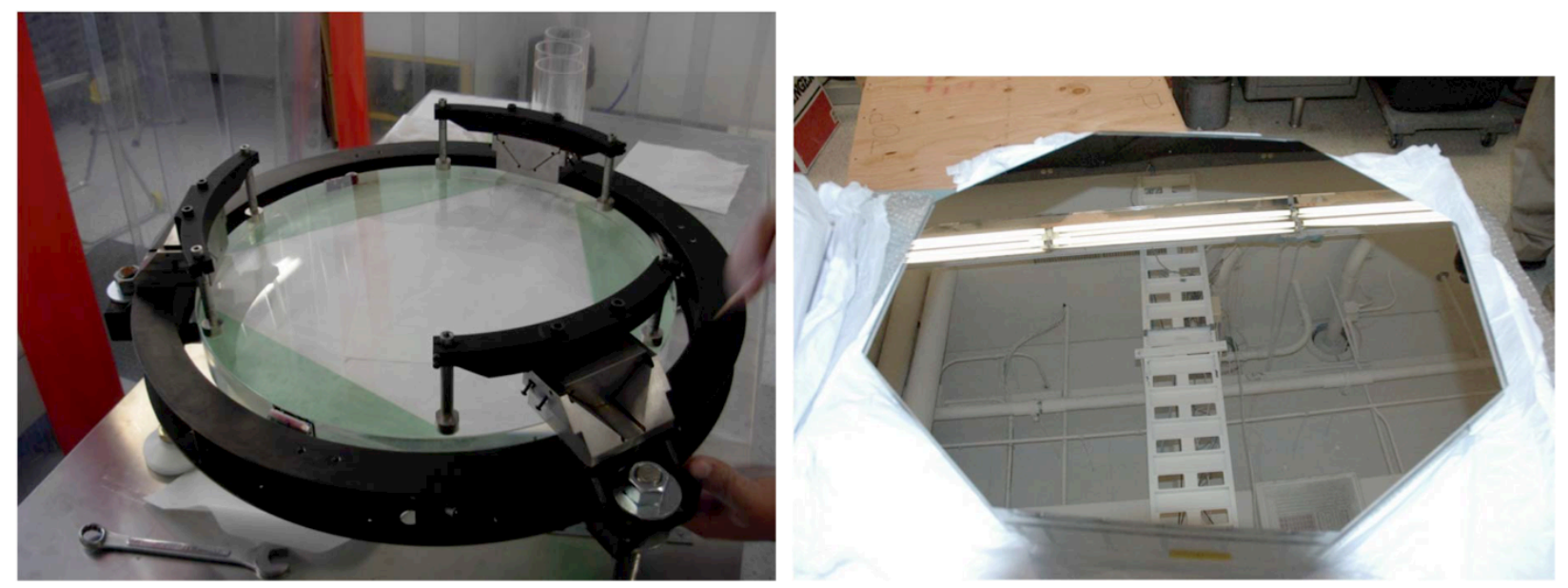

Figure 6. (Left) One of two $40 \mathrm{~cm}$ diameter plano-aspheric lenses installed in its final cell. These lenses form the exit pupil of the laser launch telescopes. The plano side also serves as a retroreflector for aligning the launch projector with an interferometer while mounted on the telescope. (Right) One of four fold flats that direct the laser beams to the sky. 
The entire light path up to the large collimating lens will be sealed inside a lightweight aluminum dust tube that will also incorporate baffles to prevent misdirected laser light from exiting the tube and creating a safety hazard. Shutters will also enclose the fold mirrors of the launch telescopes when they are not in use to preserve their reflectivity for as long as possible.

\section{WAVEFRONT SENSORS}

The returning LGS light will be detected by Shack-Hartmann wavefront sensors. ${ }^{11}$ Two WFS units, one on each side of ARGOS, will each use a single CCD to image the spot patterns of all three beacons. The WFS will be mounted to the side of the LUCIFER science instruments. Laser light reflected off the LBT's tertiary mirror and directed towards LUCIFER will be separated from the science light in the near IR by a large dichroic beam splitter coated to reflect light at wavelengths shorter than $600 \mathrm{~nm}$. Care has been taken in the design of the dichroic coating to minimize the thermal impact of a warm optic in front of the science instrument. In addition, the rear surface of the piece is cylindrical to reduce the astigmatism that arises in the converging science beam by passing through a tilted plate.

Each WFS unit comprises four main subassemblies (Figure 7). The first is a set of cameras focused at the range of the RLGS. A reflective field stop positioned at the nominal location of each beacon reflects a 1 arc min field into the corresponding patrol camera. Although these cameras are not range gated, experience with the RLGS of the MMT's GLAO system shows that the focused portion of the return beam will form a sharp image easily seen against the background of defocused laser light. The purpose of the cameras is to allow the outgoing beams to be automatically steered back into position on the field stop, and hence the WFS in the center of the field, by the control system if for any reason they become misplaced.

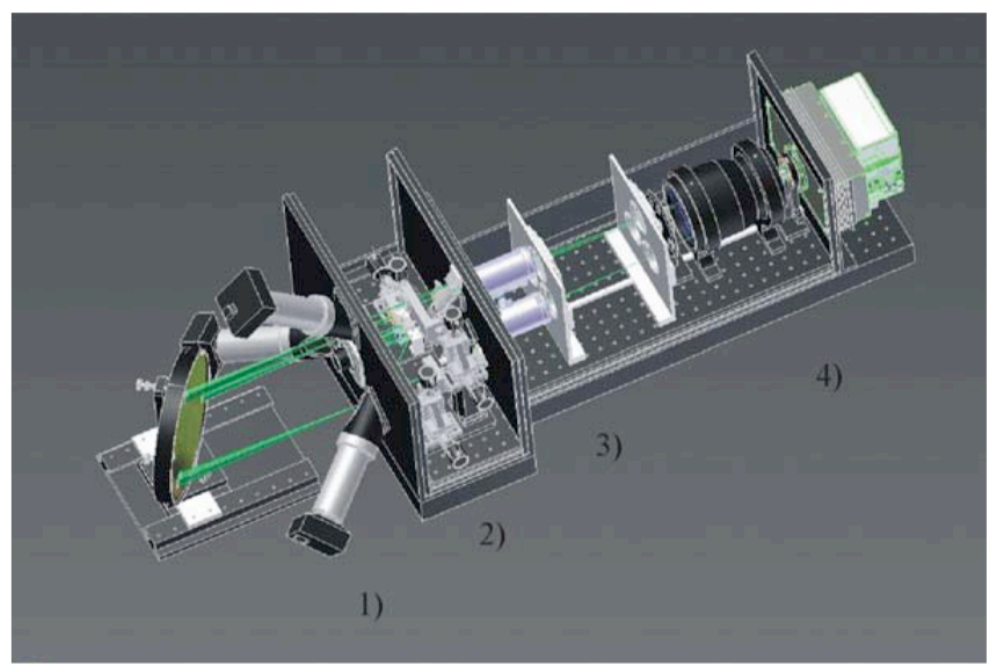

Figure 7. Schematic view of one of two WFS units designed to sense the returning light from three RLGS. The main components are: 1) field patrol cameras for beacon alignment; 2) periscope assembly to reduce the overall field angle subtended by the RLGS constellation; 3) Pockels cell range gate; 4) collimator, lenslet and detector. The entire assembly will be enclosed and flushed with dry air to prevent condensation.

Following the field stop is a set of periscopes that collapse the 2 arc min field of the RLGS constellation, allowing it all to fit onto the WFS detector at the end of the optical chain. One of the mirrors in each periscope is at a pupil and mounted on a fast steering stage. Overall beacon motion can be corrected by feedback of the mean spot centroid calculated from the corresponding WFS signals. Next are the Pockels cells, each between a pair of polarizers, that implement the RLGS range gate (Figure 8). Operation of the Pockels cells will be slaved to the laser firing signal, with an appropriate delay to accommodate the round-trip light travel time. The overall assembly has been shown in the lab to exhibit an extinction ratio of 5000 across the field of view of the stop when the cell is switched to block light. The remaining optics are common to all three beams. The pupil is imaged onto a lenslet array that puts 15 subapertures across the $8.4 \mathrm{~m}$ primary mirror, and images the RLGS spots directly onto the CCD detector. This is a $264 \times 264$ pixel pnCCD manufactured in the semiconductor laboratory of the Max Planck Institut. ${ }^{12}$ 


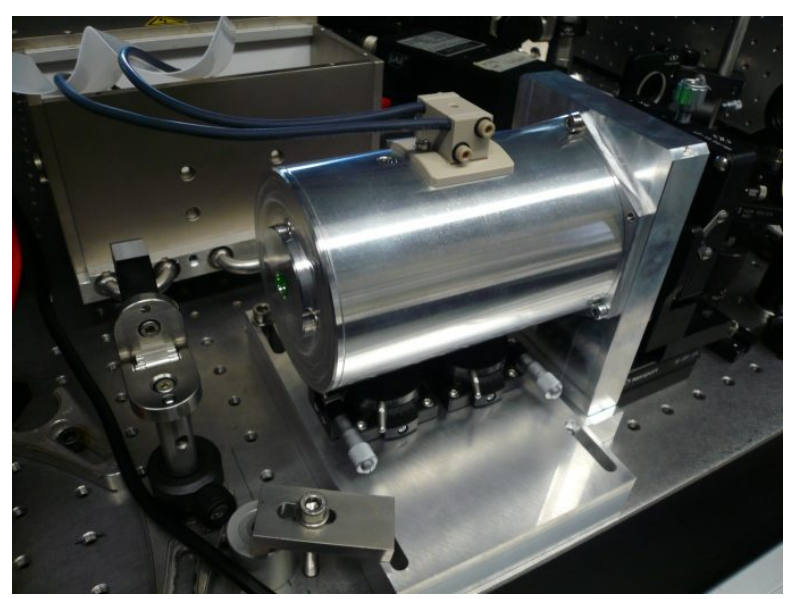

Figure 8. The first Pockels cell unit.

\section{TIP-TILT AND TRUTH WAVEFRONT SENSING}

In common with other laser-based AO systems, overall image motion in the science focal plane cannot be determined from the mean slope recorded by ARGOS' laser WFS because of unknown wander in the beams on the outbound path. Instead, it must be sensed by reference to a star within the isokinetic patch of the science object. To allow operation with the faintest possible stars, ARGOS will implement its global tilt sensors, one per eye of the LBT, with quad cell detectors based on the Excelitas SPCM-AQ4C avalanche photodiodes. The APDs themselves will be mounted in an electronics rack away from the rest of the instrument, and will be fed by optical fibers placed in a focal plane.

The tilt sensor optical fiber head will be mounted behind the dichroic beam splitter that separates the laser light, and closely mechanically coupled to the LUCIFER science instrument to minimize differential gravitational flexure. Light to feed the sensor will be reflected off the LUCIFER entrance window, which is tilted with respect to the instrument's axis, and is itself a dichroic beam splitter. In this way, the sensor will see light between $600 \mathrm{~nm}$ (the short wavelength cutoff for transmission by the laser dichroic) and $1 \mu \mathrm{m}$ (the long wavelength cutoff for reflection by the LUCIFER entrance window).

Concerned about possible quasi-static non-common path aberrations between the science focal plane and the laser WFS, which have troubled many laser AO systems, we have designed the tilt sensor feed optics also to relay a fraction of the tilt starlight to the WFS of the LBT's natural guide star AO system. ${ }^{13}$ This is a pyramid sensor which has the advantage that the number of subapertures across the pupil, and hence the spatial order of the wavefront sampling, can be dynamically defined by on-chip binning the sensor's CCD detector. By appropriate binning, and by taking long integrations, the sensor can be easily tuned to measure only those low-order aberrations that are found to drift, and with the highest signal-to-noise ratio. Corrections calculated from the output of this truth sensor will be implemented as offsets to the zero points of the RLGS WFS spot centroids.

\section{CONTROL SYSTEM}

A critical aspect of ARGOS is the software to control the system, both the real-time wavefront control servo loop and the supervisory and lower-level control functions. The basic top-level architecture defines the science instrument as the overall master of operations, communicating its needs to the telescope control system, which in turn drives ARGOS. The scheme is illustrated in Figure 9. The architecture is being implemented now on platforms running the CentOS linux operating system, with interfaces built on the ICE (Internet Communications Engine) middleware from ZeroC.

The control system is responsible for automating many procedures and implementing feedback loops that are designed to maximize the observing efficiency of ARGOS. This is of particular importance to a GLAO system. Since images are not sharpened to the diffraction limit, but rather remain somewhat blurred by turbulence in the free atmosphere, the key advantage of GLAO is a gain in observing efficiency rather than a qualitative improvement in resolution. Although the 


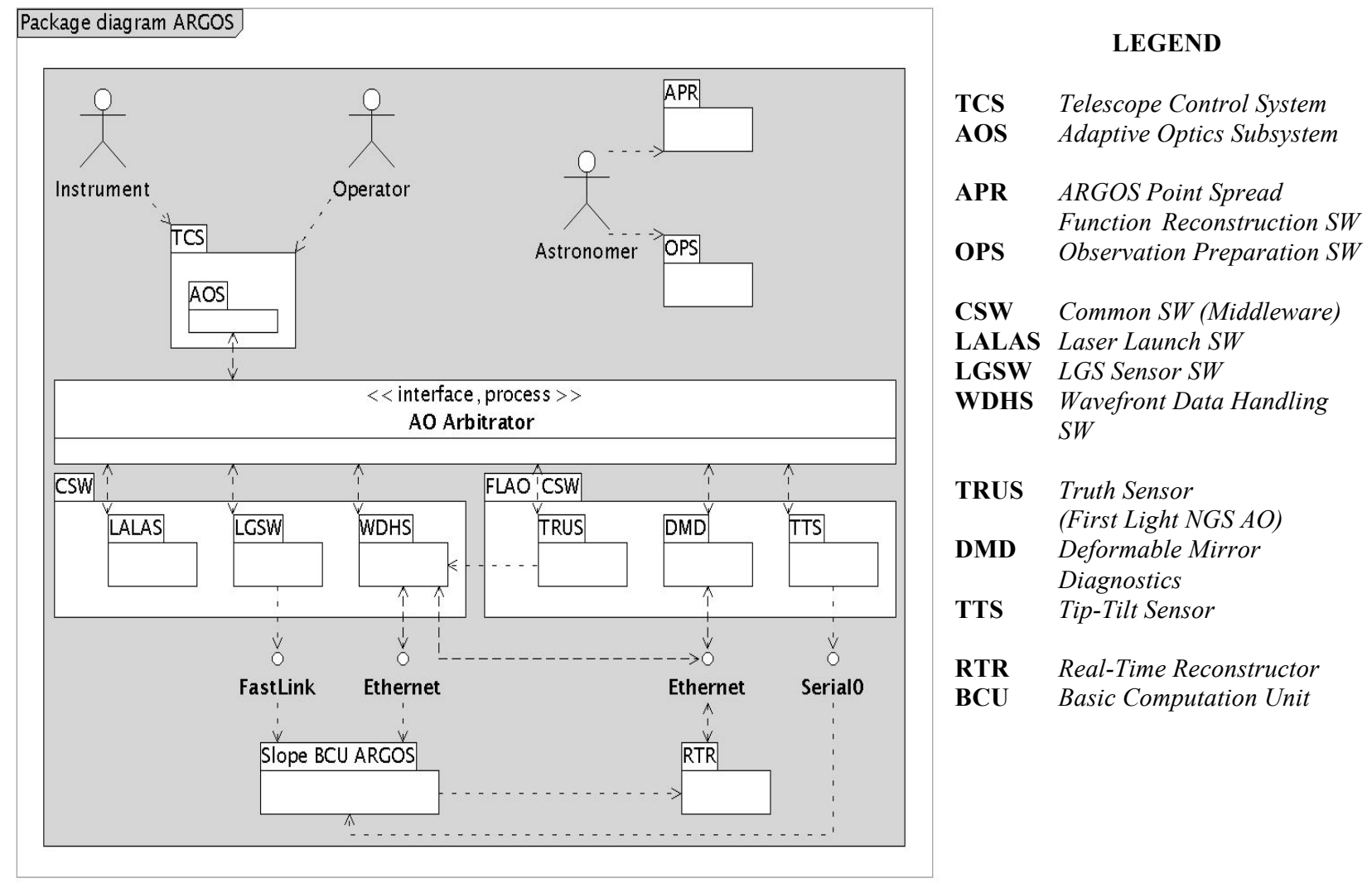

Figure 9. The overall control architecture for ARGOS. The science instrument communicates requests to the Telescope Control System, which both controls the telescope and governs the AO system. Not included in this view is the aircraft avoidance system designed to protect air traffic from accidental illumination by the lasers.

efficiency gain for ARGOS is anticipated to be as much as an order of magnitude for unresolved sources, it is of particular importance not to squander the advantage through lengthy instrument setup procedures between and during observations. The wiring diagram for the major opto-mechanical components of the system are shown in Figure 10.

\section{CALIBRATION}

Alignment, calibration, and performance tests of ARGOS must be done on the telescope. It is simply impractical to remove any of the key subsystems for testing in the lab. Furthermore, with the exception of its prime focus cameras, the LBT relies entirely on its adaptive secondary mirrors for routine operations, so removing those is not an option. Carrying out periodic calibrations on sky with starlight may be feasible but is at best an inefficient use of telescope time, and would be challenging given the uncontrolled nature of the environment. For these reasons, ARGOS is incorporating artificial stars and beacons in an optical system that may be deployed on swing-arms to the telescope's prime foci. ${ }^{14}$ These are shown in their stowed position in Figure 4. Because of the telescope's Gregorian design, it is possible to illuminate the secondaries as if with light that came from the sky and was reflected off the primary mirrors. The illumination optics will not include artificial turbulence, so closed-loop testing will be limited, but crucial calibrations such as the registration between the ASM and the WFS and the measurement of non-common path aberrations will be done during daytime operations.

Each illuminator uses an on-axis optical fiber with white light illumination to mimic starlight, and three off-axis fibers to simulate the RLGS. The on-axis fiber is mounted in an assembly at the center of each ASM (Figure 11). The remaining fibers are mounted on the swing arm in an assembly that also includes a hologram designed to mimic the off-axis aberrations of the primary mirror. The swing-arms have been manufactured from carbon fiber to minimize weight without sacrificing stiffness. They will be deployable remotely, with precision machining and preloading on the gear train to ensure that the final position is reached with the required accuracy and repeatability. 


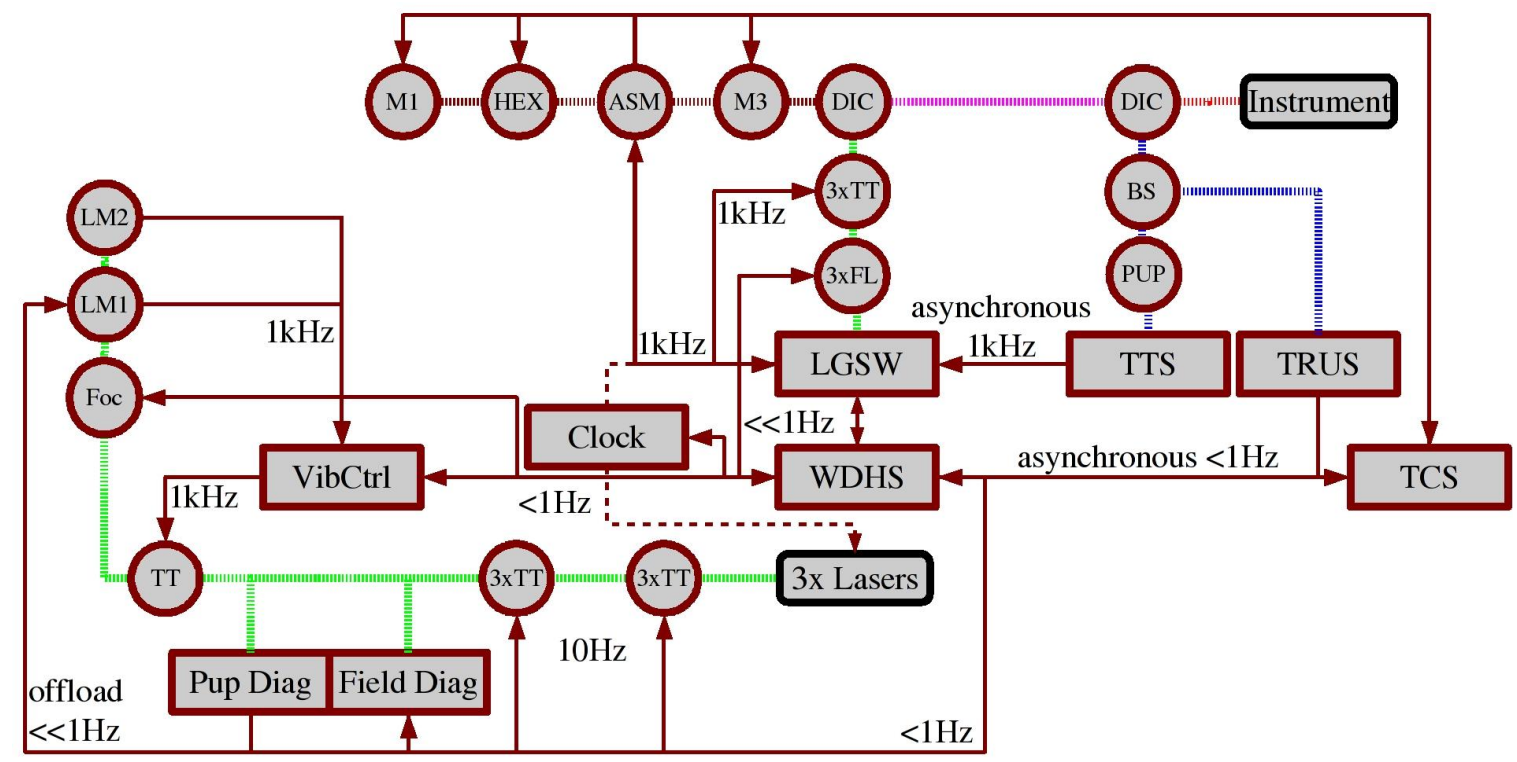

Figure 10. Overview of the control system for the opto-mechanical components of ARGOS. Blue, green, and red hashed lines represent visible, laser, and infrared light respectively. Control signals are represented by arrows, with notations for the approximate update rates. Optical elements are represented by circles, while rectangles show sensors, controllers, or other hardware. The top center represents the LBT (M1, secondary mirror hexapod, adaptive secondary, tertiary, laser dichroic). At right is the instrument (dichroic entrance window, beam splitter, steering mirror [PUP], tilt sensor, truth sensor). Lower center are the lasers, their conditioning optics and diagnostic sensors. At left is the launch telescope (vibration control, fast tip-tilt, focus stage, launch mirrors 1 and 2). In the center is the WFS (fast tip-tilt, field steering). A hard-wired clock controls the laser firing and Pockels cell switching.
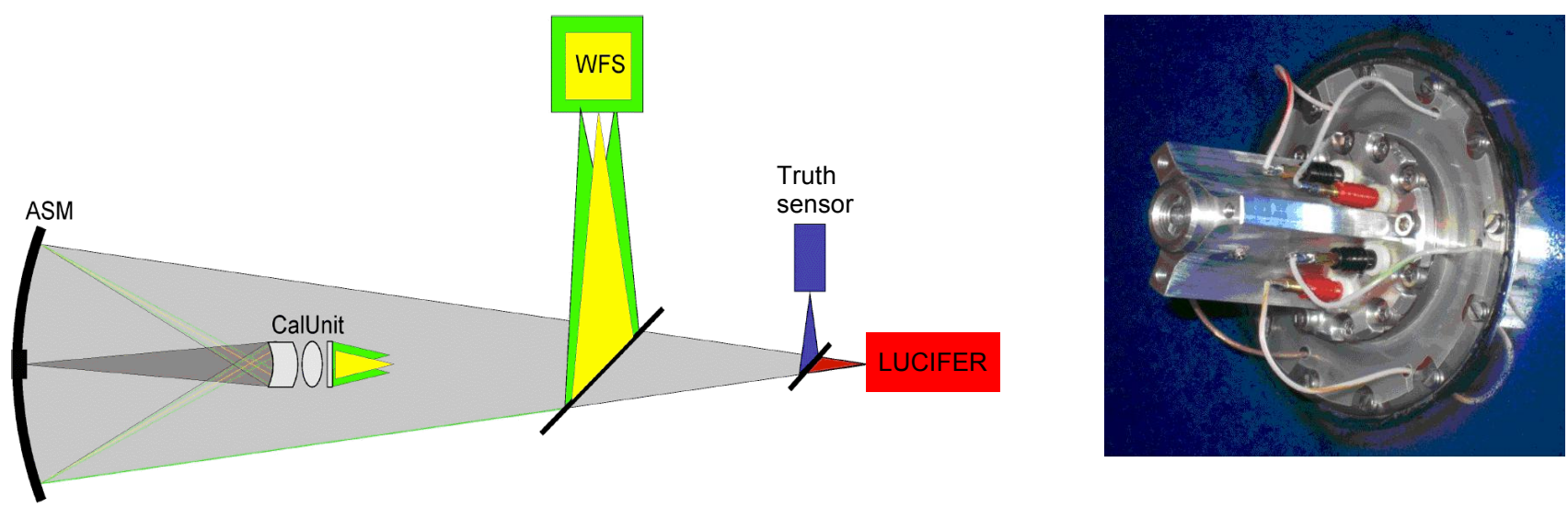

Figure 11. (Left) Cartoon of the calibration optical scheme. Light from the star simulator in the center of the ASM reflects off the front surface of the calibration unit's last lens, off the ASM, and enters the truth WFS and the science camera. Light from the RLGS simulators is transmitted through a hologram and a set of lenses, then is reflected into the WFS. (Right) The optical assembly holding the on-axis star simulator fiber attached to the central hub of one of the LBT's adaptive secondaries. The reflective surface of the ASM is opticoated blue in this picture. 


\section{AIRCRAFT DETECTION}

As with other observatories that deploy laser guide stars, the LBT must take precautions against illuminating aircraft. A system is nearing completion now, described in more detail elsewhere at this conference,${ }^{10}$ to monitor the sky and shutter the lasers in the event that an aircraft comes dangerously close to the laser beams. This precaution is particularly important for the LBT which sits in the vicinity of several civilian and military airports and directly underneath a major east-west air corridor linking Albuquerque to Tucson and southern California.

The system relies primarily on two wide-field CCD cameras, one at the top of each half of the telescope structure, with a clear view of the sky around the telescope's axis. The cameras are read approximately once a second. Differences between adjacent frames are examined for the tell-tale streaks made by aircraft lights, and the lasers are shuttered if a detection is made that appears likely to intercept the beams. As a backup, a thermal IR camera with a field of view of $10^{\circ}$ is bore-sighted with one of the LBT's two eyes. This camera runs at $30 \mathrm{fps}$, and the lasers are shuttered if any detection is made. The system is to be installed at the LBT in August 2011, ahead of most of the rest of ARGOS. This will allow us to collect significant quantities of data under normal observing conditions to quantify the impact on observing, and to design mitigation strategies before ARGOS is brought on line.

\section{SCHEDULE}

The major hardware components of ARGOS have at this point largely been acquired or manufactured. Installation of required infrastructure on the telescope has begun, with the majority of the installation targeted for spring and summer 2012. Commissioning will begin as soon as the installation is complete; the full system is expected to be ready for first light as a GLAO system at the end of that year.

\section{REFERENCES}

[1] Esposito, S. et al., "First light AO (FLAO) system for LBT: final integration, acceptance test in Europe, and preliminary on-sky commissioning results," in Adaptive Optics Systems II, eds. B. L. Ellerbroek, M. Hart, N. Hubin, \& P. L. Wizinowich, Proc. SPIE 7736, 773609 (2010).

[2] Riccardi, A. et al., "The adaptive secondary mirror for the Large Binocular Telescope: optical acceptance test and preliminary on-sky commissioning results," in Adaptive Optics Systems II, eds. B. L. Ellerbroek, M. Hart, N. Hubin, \& P. L. Wizinowich, Proc. SPIE 7736, 77362C (2010).

[3] Esposito, S. et al., "Laboratory characterization and performance of the high-order adaptive optics system for the Large Binocular Telescope," Appl. Opt. 49, G174-G189 (2010).

[4] Egner, S. E., "Multi-conjugate adaptive optics for LINC-NIRVANA: laboratory tests of a ground-layer adaptive optics system and vertical turbulence measurements at Mt. Graham," Ph.D. dissertation, University of Heidelberg, (2006).

[5] Egner, S. E. et al., "Generalized SCIDAR measurements at Mount Graham," Publ. Astron. Soc. Pac. 119, 669 (2007).

[6] Lloyd-Hart, M. and Milton, N. M., "Fundamental limits on isoplanatic correction with multiconjugate adaptive optics," JOSA A 20, 1949 (2003).

[7] De La Rue, I. and Ellerbroek, B. L., "Multiconjugate adaptive optics with hybrid laser beacon systems," in Adaptive Optics Systems and Technology II, eds. R. K. Tyson, D. Bonaccini, and M. C. Roggemann, Proc. SPIE 4494, 290 (2002).

[8] Neichel, B. et al., "The Gemini MCAO System GeMS: nearing the end of a lab-story," in Adaptive Optics Systems II, eds. B. L. Ellerbroek, M. Hart, N. Hubin, \& P. L. Wizinowich, Proc. SPIE 7736, 773606 (2010).

[9] Peter, D. "An optimized controller for ARGOS: using multiple wavefront sensor signals for homogeneous correction over the field", in Adaptive Optics Systems II, eds. B. L. Ellerbroek, M. Hart, N. Hubin, \& P. L. Wizinowich, Proc. SPIE 7736, 773615 (2010).

[10] Newman, K and Hart, M., "An automated aircraft detection system to prevent illumination from the laser guide star beacons at the MMT and LBT," these proceedings.

[11] Busoni, L. et al., "Final design of the wavefront sensor unit for ARGOS, the LBT's LGS facility", in Adaptive Optics Systems II, eds. B. L. Ellerbroek, M. Hart, N. Hubin, \& P. L. Wizinowich, Proc. SPIE 7736, 77365K (2010). 
[12] Orban de Xivry G. et al., "Wide-field AO correction: the large wavefront sensor detector of ARGOS", in Adaptive Optics Systems II, eds. B. L. Ellerbroek, M. Hart, N. Hubin, \& P. L. Wizinowich, Proc. SPIE 7736, 77365C (2010).

[13] Esposito, S. et al., "Laboratory characterization and performance of the high-order adaptive optics system for the Large Binocular Telescope," Appl. Opt. 49, G174-G189 (2010).

[14] Schwab C. et al., "Calibration strategy and optics for ARGOS at the LBT", in Adaptive Optics Systems II, eds. B. L. Ellerbroek, M. Hart, N. Hubin, \& P. L. Wizinowich, Proc. SPIE 7736, 773630 (2010). 